\title{
The effect of disorder on the fracture nucleation process
}

\author{
S. Ciliberto, A. Guarino and R. Scorretti \\ Ecole Normale Supérieure de Lyon, Laboratoire de Physique , \\ C.N.R.S. UMR5672, \\ 46, Allée d'Italie, 69364 Lyon Cedex 07, France
}

November 8, 2018

\begin{abstract}
The statistical properties of failure are studied in a fiber bundle model with thermal noise. We show that the macroscopic failure is produced by a thermal activation of microcracks. Most importantly the effective temperature of the system is amplified by the spatial disorder (heterogeneity) of the fiber bundle. The case of a time dependent force and the validity of the Kaiser effects are also discussed. These results can give more insight to the recent experimental observations on thermally activated crack and can be useful to study the failure of electrical networks.
\end{abstract}

PACS: 05.70.Ln, 62.20.Mk,61.43.-j

\section{Introduction}

Material failure is a widely studied phenomenon not only for the very important technological applications but also for the fundamental statistical aspects, which are not yet very well understood. Many models have been proposed to give more insight into the statistical analysis of failure. Among the most studied ones we can mention the fuse and the non-linear spring networks [1, 2, 3, 4, 5 , 6, 7], which can reproduce several features of crack precursors experimentally observed in heterogeneous materials subjected to a quasi-statically increasing stress [8, 9, 10, 11, 12, 13. Specifically the power law behaviour of the acoustic emission observed in several experiments close to the failure point. However the non-linear spring networks and the other related models, in their standard formulation, are unable to describe the behaviour of a material subjected to a creep-test, which consists in submitting a sample to a constant stress till the failure time. Creep-tests are widely used by engineers in order to estimate the sample lifetime as a function of the applied stress. Modified fuse networks have been proposed to explain the finite lifetime of a sample subjected to a constant stress. In ref. [A] for example, the Joule effect has been considered to explain 
delayed crack. Instead in ref. [5, 6] phenomenological force dependence of the fiber lifetime has been proposed, but not very well justified from a physical point of view. These models do not explain the recent experimental results on 2-D crystals [14, gels 15] and heterogeneous materials 16].

These experiments show that the lifetime $\tau$ of a sample, subjected to an imposed stress $P$ is well predicted by the equation

$$
\tau=\tau_{o} \exp \left(\alpha \frac{\Gamma^{d} Y^{(d-1)}}{K T_{e f f} P^{(2 d-2)}}\right)
$$

where $\tau_{o}$ is a constant, $\Gamma$ the surface energy, $Y$ the Young modulus, $K$ the Boltzmann constant $\alpha$ a constant, which depend on the geometry, $T_{\text {eff }}$ is an effective temperature and $d$ the dimensionality of the system. Eq.11 was first derived by Pomeau and Golubovic [17, 18] for $d=2$ and $d=3$. It has been generalized to any $d$ in ref. [15]. The main physical hypothesis behind eq.11 is that the macroscopic failure of a material is produced by a thermal activation of micro cracks 17, 18]. In the original Pomeau's theory [17] $T_{\text {eff }}$ of eq.11 coincides with the thermodynamic temperature $T$ while, experimentally, $T_{\text {eff }}>>T$ [14, 15. 16]. Eq.(11) is based on the idea that the sample failure is due to the thermal nucleation of one defect (microcrack) initially present inside the material 17 . However, experimentally it has been observed that the macroscopic failure is in some way related to the nucleation of many defects; this important point will be discussed at the end of this paper. Using reasonable values for the material constants in eq.116] one sees that thermal fluctuations are too small to activate the nucleation of microcraks in times $\tau$ measured in the experiments. It has been measured that the temperature needed to have the measured lifetimes $\tau$ should be of order of several thousands Kelvin. Specifically it has been estimated that for wood $T_{\text {eff }} \geq 3000 K$ [16], in $2 \mathrm{D}$ crystals $1000 K<T_{\text {eff }}<2500 K$ [14 and $T_{\text {eff }}>10^{10} \mathrm{~K}$ for gels 15 . In several materials the lifetime $\tau$ of the sample is not influenced, in the limit of experimental errors $(20 \%)$, by a variation of the temperature $T$ from 20 to $90{ }^{\circ} C$. In contrast, experiments on 2D-crystals [14 show that $\tau$ depends on $T$. Furthermore the lifetime statistics is close to Gaussian in ref. 114, 16. Thus eq.17 seems to give the right dependence on $P$ of the sample lifetime but there are a lot of experimental observations that are not completely in agreement with a standard nucleation problem. To explain these results it has been supposed [16] that the lifetime $\tau$ of the sample depends on the heterogeneity of the material and that disorder in some way enhances 1 thermal fluctuations so that the nucleation time of defects becomes of the order of the measured ones.

Motivated by these experimental observations and to check the validity of our hypothesis we proposed [19, 20] a model based on a democratic fiber bundle model (DFBM) with thermal noise. The DFBM is the simplest spring network proposed a long time ago by Pierce [21 to study cable failure. The DFBM has been studied by several authors [1, 2, 3, 22, 23, and it turns out to be a

\footnotetext{
${ }^{1}$ The term "enhances" has to be understood in the sense that the effect of the disorder is such that the system behaves like if the real temperature is bigger
} 
quite realistic model for long flexible cables, low-twist yarn and more generally for composite heterogeneous materials. In a classical DFBM, which is a deterministic model, it is not possible to observe stochastic phenomena such as nucleation. Introducing thermal noise into the system we transformed the DFBM in a stochastic model, so that processes such as nucleation become observable.

The purpose of this paper is to extend the results of our previous work [19, 20] and those obtained analytically by Roux 24]. We will show that, adding to the spring network a noise, which plays the role of a temperature, it is possible to reproduce the behavior of a material subjected to a creep-test. Furthermore we can prove that, for such a process, the noise is "amplified" by the network disorder.

The paper is organized as follows. In section 2 the modified DFBM model is introduced. In section 3 the statistical features of the model are studied analytically. Specifically we compute how failure time $\tau$ is related to the disorder of the material, when the bundle is subjected to a constant force. In section 4 the results of section 3 are verified numerically. In section 5 we study the lifetime in the general case of a bundle subjected to a time dependent force $F(t)$. We also propose a formula, which allows us to estimate the failure time $\tau$ by knowing the whole history of the medium, that is to say, the time dependence of the imposed force $F(t)$. In section 6 we give a comprehensive explication of some facts related to Kaiser effect, with a comparison between the experimental data and the numerical results obtained with our model. Discussion and conclusions follow in section 7 .

\section{The model}

We simulate the behavior of an elastic heterogeneous medium subjected to an imposed tensile force $F$ using a DFBM with thermal noise. For this purpose, we model the medium as a system of $N$ parallel elastic fibers whose extremities are fixed on a rigid support as shown in fig. 1. This model, widely studied in the quasi static regime [1, 2, 3, 过, is equivalent to $N$ springs in parallel subjected to a total tensile force $F$. Specifically we have studied the model by using the following rules:

I The external applied force $F$ produces a local force $f_{i}$ on each fiber. $F$ is democratically distributed in the net: $F=\sum_{i=1}^{N} f_{i}$.

II The local force $f_{i}$ on the i-th fiber produces a local strain $e_{i}$. Being the media elastic, force and displeacement are linked by the Hooke's law:

$$
f_{i}=Y \cdot e_{i}
$$

where $Y$ is the stifness, which is assumed to be the same for all the fibers: $Y_{i}=Y$. 
III The strength of each fiber is characterized by a critical stress $f_{c}(i)$ : if at time $t$ the $\mathrm{i}$-th fiber local stress $f_{i}(t)$ is greater than a critical stress $f_{c}(i)$ the fiber cracks, and the local force falls to zero at time $t+1$. Further, we assume that in this process some energy $\epsilon_{i}$, proportional to the square of local force $f_{i}$, is released. For sake of simplicity we will assume $\epsilon_{i}=$ $0.5 f_{i}^{2} / Y$. The critical stress $f_{c}(i)$ is a realization of a random variable that follows a normal distribution of mean $<f_{c}>$ and variance $K T_{d}$ :

$$
f_{c}(i) \sim N_{d}\left(<f_{c}>, K T_{d}\right)
$$

We call $N_{d}$ the disorder noise.

IV Each fiber is subjected to an additive random stress $\Delta f_{i}(t)$, which follows a zero mean normal distribution of variance $K T$ :

$$
\Delta f_{i}(t) \sim N_{T}(0, K T)
$$

being $t$ the time. We call $N_{T}$ the thermal noise. We assume that $\Delta f_{i}(t)$ is a white random process, which is independent in each fiber, i.e. the correlation function $E\left[\Delta f_{i}\left(t_{1}\right) \cdot \Delta f_{j}\left(t_{2}\right)\right]=0$ if $t_{1} \neq t_{2}$ or $i \neq j$. A time step of the model corresponds to the application of the noise to all the fibers. 25]

The first three items are those used in the standard formulation of the fiber bundle model. A new feature, which is similar to a thermal activation process, is introduced in $[\mathrm{IV}]$ to explain the dependence of the failure time on a constant applied stress. The model has the following properties. We see from [I] that there exists a long-range interaction between the fibers, that is, under the specified boundary conditions, the breaking of some fibers produces an increase of the local force on the other ones. Indeed if a number $n(t)$ of fibers is broken at time $t$ then the local force on each of remaining fibers is:

$$
f_{i}(t)=\frac{f_{o} N}{N-n(t)}+\Delta f_{i}(t)
$$

where $f_{o}=F / N$ is the initial force per fiber. Procedure [II] specifies the elastic behaviour of fibers. Procedure [III] models the heterogeneity of the medium: if there is no disorder noise (i.e. $K T_{d}=0$ ) all the fibers are strictly equal, and if there is no thermal activation (i.e. $K T=0$ ) the medium breaks down instantaneously when $F=N f^{(c)}$. Conversely, if $K T_{d}>0$ fibers begin to crack before the final break-down of the media. The assumption that all the disorder in the model appears in the strength distribution rather than in the elastic constants may be argued by noticing that the effective elastic constant of a single fiber is essentially the average of the local elastic constant along the fiber, while the strength is determined by its weakest point [2]. A thermal activation process is introduced in [IV] to explain the dependence of failure on time; while [I-III] are standard procedures, [IV] is new with respect to DFBM.

In this paper we want to study the failure time $\tau$ of the sample as a function of $f, K T$ and $K T_{d}$. 


\section{Some analytical results}

\subsection{The energy barrier}

Eq.11 has been obtained by Pomeau by considering that the final fracture of a material is produced by a thermally activated nucleation process of microcracks. The energy barrier of this processes has been estimated by using the Griffith criterion [26] for the stability of a fracture in an elastic medium. In this section we want to construct a similar criterion for the bundle by using the same procedure used in [17, 26]. More specifically we compute the energy barrier that the system has to overcome in order to break the bundle when $n$ fibers are broken and a total constant force $F$ is applied. We begin by noticing that the strain energy in the bundle with a constant tensile force is $U_{E}=F^{2} \lambda / 2$ where $\lambda=1 /[Y(N-n)]$ is the bundle compliance. The change in the potential energy due to the damage extension is $\Delta U_{A}=-F^{2} \Delta \lambda$. Thus the mechanical energy is $U_{M}=U_{A}+U_{E}=-F^{2} /[2 Y(N-n)]+F^{2} /(2 Y N)$. The energy $U_{f}$ used to break $n$ fibers can be computed by considering the energy needed by $n$ fibers to reach the elongation corresponding to $f_{c}$, that is $U_{f}=f_{c}^{2} /(2 Y) n$. Thus the total energy ( for $\mathrm{Y}=1$ ) is

$$
E_{t o t}=\frac{1}{2}\left[\frac{F^{2}}{N}-\frac{F^{2}}{N-n}+f_{c}^{2} n\right]
$$

At $n=n_{c}=N-F / f_{c}$ this energy has a maximum:

$$
E_{\max }=\frac{f_{c}^{2} N}{2}\left(\frac{F}{N f_{c}}-1\right)^{2}
$$

This means that for $n>n_{c}$ the force per bond is larger than $f_{c}$. Thus the bundle is unstable and will break. Following Pomeau's activation model the minimum energy per fiber $E_{\max } / N$ has to be compared to $K T$ to estimate the lifetime of the bundle. Thus the lifetime of the sample has a different functional dependence than eq.11:

$$
\tau=\tau_{o} \exp \left[\frac{f_{c}^{2}}{2 K T}\left(\frac{f}{f_{c}}-1\right)^{2}\right]
$$

where $f=F / N$. Comparing eq.11 with eq.8 we see that the functional dependence of $\tau$ on $f$ is different for the bundle (eq.8) and for a solid (eq.1). This difference is obviously due to the different geometry of the tensile force redistribution in the bundle and in the solid. In section 4 we will show, using numerical data, that eq. 8 well describes the behaviour of the lifetime of the bundle, measured in numerical simulations. However eq.8 can be obtained by a statistical analysis of the DFBM with noise. A similar analysis has been performed by Roux 24] and in the next section we extend his analysis. 


\subsection{Statistical analysis}

We describe here only the main results. The details of the calculation can be found in the appendix.

\subsubsection{The lifetime of the homogeneous fiber bundle}

We first consider the homogeneous case where the threshold force $f_{c} \equiv 1$ is the same for all of the fibers. Let $P(\eta)=\int_{\eta}^{\infty} \frac{1}{\sqrt{2 \pi K T}} \exp \left(-\frac{x^{2}}{2 K T}\right) d x$ the probability that the thermal noise has an amplitude larger than $\eta$. The mean force present on each fiber is $f=f_{o} \frac{N}{N-n}$.

It can be proved that in the limit $N \rightarrow \infty$ the ratio $\frac{n}{N}$ becomes independent of $N$ (see appendix). Thus we can introduce the function $\phi(t)$ (fraction of broken fibers at time $t)$ and the complementary function $\phi_{c}(t)$, defined as:

$$
\begin{aligned}
\phi(t) & =\lim _{N \rightarrow \infty} \frac{n}{N} \\
\phi_{c}(t) & =1-\phi(t)
\end{aligned}
$$

In the appendix we show that one can write the following equation for $\phi(t)$ :

$$
\phi(t)=\int_{0}^{t} \phi_{c}(\zeta) \cdot P\left(1-\frac{f_{o}}{\phi_{c}(\zeta)}\right) d \zeta
$$

that is:

$$
\frac{d \phi(t)}{d t}=\phi_{c}(t) \cdot P\left(1-\frac{f_{o}}{\phi_{c}(t)}\right)
$$

The failure time $\tau$ is defined as the time at which all the fibers are broken. In the appendix we show that in the limit $\phi<<1$ and $2 \sqrt{2 K T}<\left(1-f_{o}\right)$ it is possible to write an approximated solution of eq.12 with a normal distribution of the thermal noise. This solution is:

$$
\tau \simeq \frac{\sqrt{2 \pi K T}}{f_{o}} \exp \left(\frac{\left(1-f_{o}\right)^{2}}{2 K T}\right)
$$

and

$$
\phi(t) \simeq-\frac{1}{C} \ln \left(\frac{\tau-t}{\tau}\right)
$$

where

$$
C=\frac{\left(1-f_{o}\right) f_{o}}{K T}
$$

Notice that, except for logarithmic corrections, the main dependence of $\tau$ on $f_{o}$ and of $K T$ in eq.8 and in eq.13 is the same. 


\subsubsection{The lifetime of the disordered fiber bundle}

In the disordered case the breakdown threshold $f_{c}$ is a random variable. In order to find an analytical solution of $\tau$ as a function of $f$, one has to make several approximations, which are described in the appendix. When $f_{c}$ is normally distributed with mean $\left\langle f_{c}\right\rangle=1$ and variance $K T_{d}=\sigma_{o}^{2} / 2$, one finds that in the limit of $\phi<<1,2 \sqrt{2 K T}<\left(1-f_{o}\right)$ and $2 \sqrt{2 K T_{d}}<\left(1-f_{o}\right)$ a good approximation of the failure time is:

$$
\tau \simeq \tau_{o} \exp \left(\frac{\left(1-f_{o}\right)^{2}}{2 K T_{e f f}}\right)
$$

with

$$
K T_{e f f} \simeq \frac{K T}{\left(1-\frac{\sqrt{\pi} \sigma_{o}}{2\left(1-f_{o}\right)}\right)^{2}}
$$

and

$$
\tau_{o}=\frac{2 \sqrt{2 \pi K T}}{\left(f_{o}-\sqrt{\pi} \sigma_{o}\right)\left[1+\exp \left(-\frac{\sqrt{\pi} \sigma_{o}\left(1-f_{o}\right)}{K T}\right)\right]}
$$

One notices that for $K T_{d} \neq 0$ there is an effective temperature, which is an increasing function of the disorder variance $K T_{d}$. This important observation will be numerically checked in sect.4.

Another interpretation of eq.15 and eq.16 is that the disorder changes the critical force from $f_{c}=1$ (in the ordered case) to a smaller value $\left(1-\sqrt{\pi} \sigma_{o} / 2\right)$ in the disordered case. We consider that the interpretation in terms of $K T_{\text {eff }}$ is the best one. Indeed one can show (see 24|)that if the dynamics would be dominated by the fibers with thresholds in the tails of $G_{o}\left(f_{c}\right)$ than $K T_{\text {eff }}=K T+K T_{d}$. The interpretation given in eq.16 stresses the difference between a tail dominated dynamics and a dynamics dominated by the fibers with $f_{c}>f_{\min }$.

\section{Numerical results}

In this section we check the analytical results obtained in sect. 3 on a numerical simulation on the fiber bundle. In the following we will assume without loss of generality $f_{c}=1$ and $N=1000$. We have checked that the results do not change considerably up to $N=10^{6}$. For each values of the parameters $K T, F$, $K T_{d}$ we have repeated the experiments at least 10 times in order to estimate the scattering of the results in different realizations. 


\subsection{Failure time at constant force}

\subsubsection{The homogeneous case}

In order to study the behaviour of failure time $\tau$, we started by imposing a force $F(t)=F$ constant in time (creep test). If disorder noise and thermal noise are both zero (i.e. $K T=K T_{d}=0$ ), the system either breaks at time $\tau=0$ only if $F \geq N f_{c}$ or it does not break at all otherwise. Conversely, if thermal noise is not zero (i.e. $K T \neq 0$ ), we observe (fig. 2 a) that failure time $\tau$ has an exponential dependence on $\left(1-f_{o}\right)^{2}$ for any fixed value of $K T$, specifically:

$$
\tau \sim \exp \left[\alpha\left(1-f_{o}\right)^{2}\right]
$$

where $\alpha$ is a fitting parameter, which is a function of $K T$. Furthermore at constant $f_{o}$ (see fig. $2 \mathrm{~b}$ ), the failure time $\tau$ depends on $K T$ as follows:

$$
\tau \sim \tau_{o} \exp \left(\frac{A}{K T}\right)
$$

where $A$ is a function of $f$. We notice that these results are similar to those predicted by the activation model discussed in section 3 and that the lifetime is well described by eq.8. More precisely one can check the predictions of eq.13. by plotting in fig.2 $\log \left(\frac{\tau f_{o}}{\sqrt{2 \pi K T}}\right)$ as a function of $\frac{\left(1-f_{o}\right)^{2}}{2 K T}$. We see that all data collapse on a single straight line of slope 1, verifying in this way the hypotheses made to obtain eq.13. In fig. 3 we plot the time evolution of $\Phi$ as a function of time. The continuous line is the numerical solution of eq.12. The circles correspond to the mean values obtained in several direct numerical simulations of the DFBM. In the inset we compare the result of the direct numerical simulation with the approximated solution (eq.14) of eq.12.

Fig.2 and fig. 3 show that the hypotheses made to obtain eq.13 and eq.14 are well verified by the numerical integration of the model.

\subsubsection{The heterogeneous case}

We are now interested in studying the dependence of the failure time $\tau$ on disorder noise. To this aim, we have done simulations keeping $K T_{d}$ fixed at constant (non-zero) values. In fig. 1 we plot the dependence of $\tau / \sqrt{2 \pi K T}$, at $f_{o}=0.54$, as a function of $1 / K T$ for different values of $K T_{d}$. We notice that for the same values of $K T, \tau$ decreases by several order of magnitude by increasing the disorder noise. Most importantly, we see that for any values of $K T_{d}$ the leading dependence of $\tau$ on $K T$ is the same of the ordered case (eq.19), but the value of $A$ and $\tau_{o}$ depend on $K T_{d}$. Specifically, increasing $K T_{d}$ the value of $A$ decreases whereas $\tau_{o}$ increases. Using eq.15 and eq.16 one obtains that $A$ in the heterogeneous case is:

$$
A=0.5\left(1-f_{o}\right)^{2}\left(1-\frac{\sqrt{\pi} \sigma_{o}}{2\left(1-f_{o}\right)}\right)^{2}
$$


From the best fits of the numerical data of fig. A one can measure the values of $A$ as a function of $f$ and $K T_{d}$. The prediction of eq. 20 can be accurately checked by plotting $2 A /\left(1-f_{o}\right)^{2}$ as a function of $\sqrt{2 \pi K T_{d}} /\left(1-f_{o}\right)$. In fig 5 we clearly see that the numerical values of $2 A /\left(1-f_{o}\right)^{2}$, obtained for different values of $f_{o}$, collapse on the same curve, which agrees quite well with the theoretical prediction, continuous curve, obtained using eq.2q.

The dependence of $\tau$ on $K T_{d}$ is plotted in fig. 6 for several values of $f$ and $K T$. The continuous lines are the predictions of eq.15. We see that the main dependence of $\tau$ on $K T_{d}$ is very well described by eq.15. The deviation at large $K T_{d}$ are due to the approximations made to get the analytical expression eq.15. This equation and numerical results show that $\tau_{o}$ increases as a function of $K T_{d}$. Therefore the observed reduction of the sample lifetime for increasing $K T_{d}$ is mainly due to a decrease of $A$. In looking at fig. 4 and from eq.15 we also notice that the derivative of $\tau$ with respect to $K T$ decreases as a function of $K T_{d}$. Summarizing, the following conclusions can be extracted from the numerical and the analytical results:

B1 The failure time $\tau$ decreases as the disorder noise variance $K T_{d}$ increases, that is the more the medium is heterogeneous, the smaller the failure time is, see fig.t.

B2 As the disorder noise variance $K T_{d}$ increases, the derivative of $\tau$ with respect to $K T$ decreases, that is failure time $\tau$ becomes less sensitive to the effective value of the thermal noise variance, as shown in fig. 4 .

Thus the role of the spatial disorder is that of multiplying $K T$ by a factor, which is an increasing function of $K T_{d}$. This is equivalent to say, as we already mentioned in section 3, that an approximation of $K T_{\text {eff }}$ is given by eq.16. Furthermore fig. 1 and fig. 6 show that the disorder reduces the dependence of $\tau$ on the variation of the temperature $T$.

\subsection{Comparison with experimental results}

These results allow us to give a reasonable explanation of the experimental observations reported in the introduction. In fact, experiments show that the lifetime $\tau$ of very heterogenous materials is independent of $T$, 16 , while the lifetime $\tau$ of quite homogenous materials as 2D-crystals depends on temperature $T \mid 14$. This is consistent with the numerical results of our model. As shown in fig. 1 when $K T_{d}$ is small as in 2D-crystals the dependence of $\tau$ on $K T$ remains important, whereas this dependence becomes negligible when $K T_{d}$ is huge (like in chip-board panel wood and fiberglass). In any case disorder noise induces an effective temperature much larger than the thermodynamic one $T$.

\subsection{Failure time distribution}

We have studied the distribution of failure time $\tau$ when the parameters $K T$ and $F$ are constants, in the case of a single fiber and in the case of a net of many 
fibers. Clearly, in the case of a single fiber the probability of failure is constant in time, so that $\tau$ follows a Poisson's law fig. 7 . Conversely, in the case of a net of fibers one sees that $\tau$ is normally distributed. This result is coherent with experimental results, which show that in highly disordered materials and large aspect ratio the sample lifetime distribution is Gaussian.

\section{Generalization to a time dependent imposed force}

In ref. 16. we proposed to generalize eq.8 such that it may be applied to any time dependent pressure; a similar procedure is proposed here. Suppose that $\frac{1}{\tau(F)}$ is the damage density per unit time of the medium subjected to a force $F$. For an arbitrary load $F(t)$ the total damage density at time $t$ is:

$$
I(t)=\int_{0}^{t} \frac{1}{\tau_{0}} \exp \left[-\frac{\left(1-F\left(t^{\prime}\right) / N\right)^{2}}{K T_{\text {eff }}}\right] d t^{\prime}
$$

thus the certitude of failure is attained when $I(t)=1$. Notice that this yields eq. 8 if $F(t)=F$.

In order to verify whether this equation holds, we have done simulations imposing $F(t)$ with different dependencies on time.

As an example we consider a linear dependence on time of $F: F=M t$. Inserting this linear dependence in eq.21 we can approximately compute the dependence of $\tau$ on the slope $M$ getting for $M<<\sqrt{\frac{2 K T}{\pi}}$ :

$$
\tau=\frac{\left(1-\sqrt{2 K T} \delta_{M}\right)}{M\left(1+0.5 \sqrt{2 K T} / \delta_{M}\right)}
$$

where

$$
\delta_{M}=\ln \left(\sqrt{\frac{2 K T}{\pi}} \frac{1}{M}\right)
$$

In fig. 8 we plot $\tau$ as a function of $M$ at $K T=0.01$. Circles are the computed values and the continuous line is the prediction of eq. 22. The agreement between theoretical prediction and numerical simulations is quite good.

Further we apply to the bundle a sinusoidal force as shown in fig. 9 . In this figure the dotted line is the external applied force and the dashed line represents the numerical integration of the integral in eq.21. The circles indicate the occurrence of breaking events. Finally the dotted line is the fraction of broken fiber $\phi$ as measured by the direct numerical simulation of the fiber bundle. We clearly see that the lifetime predicted by eq.21 perfectly agrees with that of the direct numerical simulation. This shows that eq.21 gives a good estimate of the breaking time for a periodic forcing. 


\section{Kaiser effect}

In 1950 Kaiser discovered that the acoustic emission of a stressed metal sample is zero if the applied stress is smaller than the previously applied maximum 28. This effect, usually called Kaiser effect, was also discovered in rock materials 29, 30], but its existence was seriously questioned for the Westerley granite 31] and for heterogeneous materials as wood and fiberglass 11. In our simulation we call event the simultaneous breaking of several fibers, and size of the event the number $s(t)$ of fibers which break. The energy $\epsilon$ associated to an event is the sum of the energies released by the fibers that crack, that is :

$$
\epsilon=\frac{s(t)}{2 Y}\left(\frac{F}{N-n(t)}\right)^{2}
$$

The potential energy of a fiber that breaks is proportional to its accumulated elastic potential energy. If we assume that the acoustic energy is proportional to this potential energy then $\epsilon$ may be compared to the acoustic energy measured in the experiments [10, 11]. The cumulative energy $E_{a}(t)$ is the sum of $\epsilon$ from 0 to $t$.

In order to check the behaviour of our model with respect to the Kaiser effect we have imposed cyclic forces $F=F_{0}\left[1-\cos \left(\frac{2 \pi}{T_{\omega}} t\right)\right]$ to the net (the continuous line in fig. 10a). If $K T=0$, i.e. in a classic DFBM, the Kaiser effect is strictly valid: no events appear after the first period. Conversely, if $K T>0$, events (spots on fig. 10a) are produced after the first. In fig. 10 b), the number of events $N_{i}$ (continuous bars) and the energy $E_{i}$ (dashed bars) released during the i-th cycles are plotted, normalized to the values of the first cycle, as a

function of the cycle number $i$. The released energy $E_{2}$ and the number of events $N_{2}$ occurred during the second cycle are not negligible as they represent about the $50 \%$ of $E_{1}$ and $N_{1}$. From the second cycle $(i \geq 2), N_{i}$ and $E_{i}$ grow with $i$. During the fourth cycle the energy released $E_{4}$ becomes bigger than the energy $E_{1}$ released during the first cycle. The number of events $N_{i}$ grows slower than the released energy $E_{i}$ so that the average energy $E_{i} / N_{i}$ released by an avalanche during the i-th cycle grows as a function of $i$. The behaviour of $E_{i} / N_{i}$ is represented by circles on fig. 10b). The rates of the $N_{i}$ and $E_{i}$ after the first cycle depend on $K T$ and $K T_{d}$. The statistic of events is very similar to experimental observations in heterogeneous materials [11]. In conclusion, we have shown that the Kaiser effect is valid only if the thermal noise is negligible. Conversely, if a thermal noise is present the Kaiser effect is not observed.

\section{Conclusions and discussion}

To simulate the failure of heterogeneous materials, we used a DFBM with thermal noise. We observe that the breakdown of the system occurs through the coalescence of many microcracks which are driven by the thermal fluctuations and which appear at different times. Thus the lifetime $\tau$ of the DFBM with thermal noise follows a law, which is similar to an activation processes. The 
main goal of the simulation was to check the role of the disorder on the lifetime of the system. We have shown both numerically and analytically that the most important effect of the disorder is that of producing an effective temperature of the system, which is larger than that of the thermal noise. This effective temperature (eq.16) is equal to the thermal noise temperature multiplied by a factor, which is an increasing function of the disorder variance. This important result is independent on the specific distribution of thresholds. In this sense we can say that the disorder "amplifies" the thermal noise temperature. It is important to stress that that thermal noise and disorder play different roles: indeed it is the thermal noise, which drives the cracking process, while the disorder just enhances its effect. Two important hypotheses have been done to get this result. The first is that the threshold distribution evolves as a function of the fraction of broken bonds. The second is that the tails of the distribution do not play an important role in the fracture process. To estimate $\tau$, one has to consider only the part of the distribution containing about $90 \%$ of the unbroken bonds. The effective temperature of eq.16 is quite different from the result recently obtained by Roux 24. He finds that the effective temperature is the sum of $K T$ and $K T_{d}$. Indeed our result is not directly comparable with that of Roux because he estimates the time needed to cut the first fibers. This time is negligible with respect to the total lifetime of the bundle. Furthermore the number of bonds contained in the tails does not affect the resistance of the system (for example in the case of the Gaussian only $2 \%$ of the bonds have a threshold in the tails).

Our results not only demonstrate that the disorder enhances the role of thermal noise but eq.16 and fig. A clearly show that when the disorder noise variance $K T_{d}$ increases, the absolute value of the derivative $\frac{d \tau}{d K T}$ decreases, that is the failure time $\tau$ becomes less sensitive to variation of the effective value of thermal noise (not zero as well). The conclusion, that in an activation processes disorder noise induces an effective temperature larger than the thermodynamic one, has been reached for other disordered systems such as foams [32]. This kind of disorder induced enhancement of the thermodynamic temperature is an interesting result because it could be be a quite general property of disordered systems, where a thermal activated processes with long range correlation is present.

It is interesting to compare the results of our model with the recent experimental observations on the lifetime of samples submitted to a constant applied stress. It has been shown that in wood [16], fiberglass [16, gels [15] and 2Dcrystals 14] the dependence of the lifetime on the applied stress is well predicted by the Pomeau model on the delayed fracture (see eq.11). However we already mentioned in the introduction that in all of the experiments the estimated temperature was higher than the thermodynamic one and in ref. 16] we argued that the heterogeneity of the material may enhance the role of temperature. The theoretical and numerical results of this paper give new insight to this argument. The fiber bundle and the Pomeau model have a different dependence on the applied load (compare eq.8 and eq.17). This difference comes from the fact that the energy barrier is different in a real crack and in the bundle. Thus we 
neglect this difference and we focus on the role played by the disorder. The Pomeau model is based on the idea that failure of the sample is due to the thermal nucleation of one defect (microcrack) initially present inside the material [17. In contrast, this model and experiments show that the failure is due to the coalescence of many microfractures appeared at different times. We think that a good theoretical model to describe the dynamic of the DFBM and various real materials submitted to an external force could be made transforming in a statistical model the one of Pomeau. In that model the nucleation of a microcrack is the elementary process, i.e. the sample break when a certain number of microcraks have nucleated. In this case, the parameters $Y, \Gamma$ and $T_{\text {eff }}$ of eq.1, become average parameters. The strong time-dependent fluctuations of the internal forces induced by the heterogeneity (defects, microcraks ...), can be considered as a sort of noise so that, in our statistical model, $T_{\text {eff }}$ must depend on the thermal temperature but also on the disorder in the medium. As we have already mentioned our numerical and analytical results on the fiber bundle also show that the dependence on the lifetime on temperature decreases when the disorder increases. These results could explain why in very disordered materials, such as wood and fiberglass, the lifetime $\tau$ seems to be independent of temperature while $\tau$ strongly depends on temperature in weak heterogeneous materials such as $2 \mathrm{D}$-crystals.

The comparison of the lifetime of the bundle with those of real materials merits a special comment. Indeed in the bundle we consider as unit of the time a complete update of all the fibers. One can assume that the characteristic time in a real material is the time needed to redistribute the stress after that a microfracture is occurred. This time, which is given in a first approximation by the size of the sample divided by the sound speed, is of the order of $10^{-5} s$ 16. This means that the largest observed experimental time correspond to roughly $10^{10}$ time steps of the bundle. This very large $\tau$ are reached for very small $K T$ and $K T_{d}$ where our approximation becomes more precise. Thus we may assume that the comparison of this theoretical results with experiment could be rather realistic.

In the last part of the paper we have introduced the concept of density of damage per unit time finding an equation (eq.21), which predicts the lifetime $\tau$ of the DFBM for any time dependent imposed force. These numerical results agrees with experiments on wood and fiberglass 16 . Finally, we have shown that the validity of Kaiser effect is linked to the value of the thermal noise $K T$ and of the disorder noise $K T_{d}$.

Acknowledgement Part of this work has been done within a SOCRATES exchange program of the European Community between ENSL and "Facoltá d'Ingegneria, Univeristá di Firenze, Italy ". One us (R.S.) thanks "Le laboratoire de Physique de l'E.N.S.L." for the very kind hospitality during his visit.

\& Actual address: Ecole Centrale de Lyon, 69131 Ecully, France. 


\section{References}

[1] H.J Hermann, S. Roux, Statistical models for the fracture of disordered media, North-Holland, Amsterdam (1990).

[2] C. Hemmer. A. Hansen, Journ. of Applied Mech., 59, Dec (1992).

[3] J. V. Andersen, D. Sornette, K. Leung, Phys. Rev. Lett. 78, 2140 (1997).

[4] D. Sornette, K. Vanneste, Phys. Rev. Lett. 68, 5 (1992).

[5] B.D. Coleman, J. Appl. Phys., 29, 968 (1958).

[6] S.L.Phoenix, L. J. Tierney, Eng. Fract. Mech., 18, 193 (1983).

[7] Zapperi S., Vespignani A. and Stanley H.E., Nature, 338, 658 (1997).

[8] D. A. Lockner, The role of acoustic emission in the study of rock fracture, Int. J. Rock Mech. Min. Sci. \& Geomech. Abstr., 30, 883-899, (1993).

[9] J.C. Anifrani, C. Le Floc'h, D. Sornette, B. Souillard, Universal log-periodic correction to renormalization group scaling for rupture stress prediction from acoustic emissions, J. Phys. I France 5, 631-638 (1995).

[10] A. Garcimartín, A. Guarino, L. Bellon and S. Ciliberto, Statistical Properties of fracture precursors, Phys. Rev. Lett. 79 17, 3202-3205 (1997). 62

[11] A. Guarino, A. Garcimartín and S. Ciliberto, An experimental test of the critical behavior of fracture precursors, European Physical Journal B, 6, 13-24 (1998).

[12] A. Petri, G. Paparo, A. Vespignani and M. Costantini, Experimental evidence for critical dynamics in microfracturing processes, Phys. Rev. Lett. 73, 25 (1994).

[13] C. Maes, A. Van Moffaert, H. Frederix and H. Strauven, Criticality in creep experiments on cellular glass, PR B, vol 57, 9 (1998).

[14] L. Pauchard and J. Meunier, Instantaneous and time-lag breaking of a twodimensional solid rod under a bending stress, Phys. Rev. Lett. vol. 70, 23, (1993).

[15] D. Bonn, H. Kellay, M. Prochnow, K. Ben-Djemiaa and J. Meunier, Delayed fracture of an inhomogeneous soft solid, Sciences, vol. 280, pp 265267 (1998).

[16] A. Guarino, A. Garcimartín and S. Ciliberto, Failure time and microcrack nucleation, Europhysics Letters, 47, 456 (1999).

[17] Y. Pomeau, Brisure spontanée des cristaux bidimensionnel courbés, C. R. Acad. Sci. Paris, vol. 314 II, 553-556 (1992). 
[18] Gobulovic L. and Feng S., Pys. Rev. A, 40, 5223 (1991).

[19] A. Guarino, R. Scorretti, S. Ciliberto, cond-mat/9908329

[20] A. Guarino, Phd Thesis, Ecole Normale Supérieure de Lyon, France

[21] F.T. Pierce, J. Textile Industry, (1926).

[22] A. Hansen and P.C. Hemmer, Criticality in fracture: the burst distribution. Trend in Stat. Phys. (1994).

[23] M. Kloster, A. Hansen and P.C. Hemmer, Burst avalanches in solvable models of fibrous materials, cond-matt/9703170, 18 Mar (1991).

[24] S. Roux, Phys. Rev. E 62, 6164 (2000)

[25] A special comment has to be done on the source of the noise from a physical point of view. The simplest way of understanding this point is to remind that the DFBM has a one to one correspondence with a fuse network driven at imposed current. As a fuse is an electrical resistance the source of noise is the Nyquist noise on the resistance. In the case of the springs one can think that the springs have a damping, which is a source of thermal noise because of the fluctuation dissipation theorem.

[26] B. Lawn, Fracture of brittle solids, Cambridge University Press 1993.

[27] A. A. Pollock, Acoustic emission amplitude distributions, Int. Advanced in Nondestructive Testing, vol 7, pp. 215-239 (1981).

[28] J. Kaiser, PhD thesis, Tech. Hosch. Munchen, Munich (1950).

[29] K. Kurita and N. Fujii, Geophysical Res. Lett. 6, 9 (1979).

[30] T. Kanagawa, M. Haiashi, H. Nakasa, Estimation of the spatial geo-stress component in rock samples using the Kaiser effect of acoustic emissions, Tech. Report, C.R.I.E.P.I., E 375004 (1976).

[31] C. Sondergeld and L. Estey, J. Geophysics. Res, 86,2915 (1981).

[32] Sollich P., Phys. Rev. E, 58, 1, 738 (1998). 


\section{APPENDIX}

\section{Analytical results}

\section{A The homogeneous case}

Consider the homogeneous case where the threshold force $f_{c}$ is the same for all of the $N$ fibers composing the bundle. Let

$$
P(\eta)=\int_{\eta}^{\infty} G_{T}(x) d x=\int_{\eta}^{\infty} \frac{1}{\sqrt{2 \pi k T}} \exp \left(-\frac{x^{2}}{2 k T}\right) d x
$$

be the probability that the thermal noise has a fluctuation larger than $\eta$. If $n$ is the number of broken fibers at time $t$ then the force present on each fiber is $f=f_{o} \frac{N}{N-n}$. Thus the probability that a single fiber breaks in a time step is:

$$
p_{1}=P\left(f_{c}-f\right)=P\left(f_{c}-\frac{f_{o} N}{N-n}\right)
$$

The expected number of fibers that break in a time step is:

$$
R_{N}=(N-n) \cdot p_{1}=(N-n) \cdot P\left(f_{c}-\frac{f_{o} N}{N-n}\right)
$$

Notice that if this expression is smaller than one (i.e. $R_{N}<1$ ) one may think of $\langle t\rangle=\frac{1}{R_{N}}$ as the expected time between two consecutive microcracks.

These expressions allow us to get several analytical results. Indeed it can be easily proved that as $N \rightarrow \infty$ the ratio $\frac{n}{N}$ becomes independent of $N$ :

$$
\begin{aligned}
\frac{n_{t}}{N} & =\frac{1}{N} \sum_{i=1}^{t} R_{N}(i) \\
& =\frac{1}{N} \sum_{i=1}^{t}\left(N-n_{i}\right) \cdot P\left(f_{c}-\frac{f_{o} N}{N-n_{i}}\right)= \\
& =\sum_{i=1}^{t}\left(1-\frac{n_{i}}{N}\right) \cdot P\left(f_{c}-\frac{f_{o}}{\left(1-\frac{n_{i}}{N}\right)}\right)
\end{aligned}
$$

We can introduce the function $\phi(t)$ (fraction of broken fibers at time $t$ ) and the complementary function $\phi_{c}(t)$, defined as:

$$
\begin{gathered}
\phi(t)=\lim _{N \rightarrow \infty} \frac{n_{t}}{N} \\
\phi_{c}(t)=1-\phi(t)
\end{gathered}
$$


Turning the sum in eq.A.6 into an integral one can write the following equation for $\phi(t)$ :

$$
\phi(t)=\int_{0}^{t}(1-\phi(\zeta)) \cdot P\left(f_{c}-\frac{f_{o}}{1-\phi(\zeta)}\right) d \zeta
$$

that is:

$$
\frac{d \phi(t)}{d t}=(1-\phi(t)) \cdot P\left(f_{c}-\frac{f_{o}}{1-\phi(t)}\right)
$$

The failure time $\tau$ is defined as the time at which all of the fibers are broken. It can be expressed in terms of $\phi(t)$ by the condition:

$$
\phi(\tau)=1
$$

In the homogeneous case we assume $f_{c} \equiv 1$ for all of the fibers. Unfortunately no exact solution of A.10 is known, so one has to make some approximations to get an analytical result. The crucial point is that in the direct numerical simulation of DFBM one observes that $\phi \ll 1$ for most of the time: this allows us to write $\frac{1}{1-\phi} \simeq 1+\phi$ and to approximate $P(\eta)$ using an asymptotic development:

$$
P(\eta)=\frac{1}{2}\left(1-\operatorname{erf}\left(\frac{\eta}{\sqrt{2 k T}}\right)\right) \simeq \sqrt{\frac{k T}{2 \pi}} \frac{1}{\eta} \exp \left(-\frac{\eta^{2}}{2 k T}\right)
$$

Thus one can write:

$$
\begin{aligned}
\frac{d \phi}{d t} & \simeq \sqrt{\frac{k T}{2 \pi}} \frac{1-\phi}{\left(1-\frac{f_{o}}{1-\phi}\right)} \exp \left(-\frac{\left(1-\frac{f_{o}}{1-\phi}\right)^{2}}{2 k T}\right) \\
& \simeq \sqrt{\frac{k T}{2 \pi}} \frac{1-\phi}{1-f_{o}-f_{o} \phi} \exp \left(-\frac{\left(1-f_{o}-f_{o} \phi\right)^{2}}{2 k T}\right)
\end{aligned}
$$

being $f_{o} \phi \ll 1$, one may write:

$$
\begin{aligned}
\frac{d \phi}{d t} & \simeq \sqrt{\frac{k T}{2 \pi}} \frac{1}{1-f_{o}} \exp \left(-\frac{\left(1-f_{o}\right)^{2}}{2 k T}\right) \exp \left(\frac{2\left(1-f_{o}\right) f_{o} \phi}{2 k T}\right) \\
& =B \exp (C \phi)
\end{aligned}
$$

being $B$ and $C$ defined as:

$$
\begin{aligned}
B & =\sqrt{\frac{k T}{2 \pi}} \frac{1}{1-f_{o}} \exp \left(-\frac{\left(1-f_{o}\right)^{2}}{2 k T}\right) \\
C & =\frac{\left(1-f_{o}\right) f_{o}}{k T}
\end{aligned}
$$


At this point one turns to the integral equation:

$$
\int \exp (-C \phi) d \phi=B \int d t
$$

The last equation can be integrated:

$$
\frac{\exp (-C \phi)}{C}=-B t+\frac{1}{C}
$$

where the initial condition is $\phi(0)=0$.

We verified numerically that this equation describes the system behaviour for small $\phi$. Moreover one finds that as $\phi$ becomes big enough, say $\phi_{\text {break }} \simeq 0.1$, the system undergoes a cascade of microcracks until the final breakdown, which occurs after a little time. Eq. A.16) seems to represent closely the system's behaviour for almost all the time.

Thus one can write the following estimation for the failure time $\tau$ :

$$
\tau \simeq \frac{1}{C B}=\frac{\sqrt{2 \pi k T}}{f_{o}} \exp \left(\frac{\left(1-f_{o}\right)^{2}}{2 k T}\right)
$$

This expression of $\tau$ agrees with both numerical simulations and theoretical results of [24].

Using (A.17) into (A.16) one finds:

$$
\begin{gathered}
\frac{\tau-t}{\tau}=\exp (-C \phi) \\
\ln \left(\frac{\tau-t}{\tau}\right)=-C \phi
\end{gathered}
$$

Finally one finds:

$$
\phi(t)=-\frac{1}{C} \ln \left(\frac{\tau-t}{\tau}\right) .
$$

We verified (see fig.3) that these equations agree very well with numerical data, actually even for values of $\phi$ comparable to 1 . From eq A.18 one obtains that $t \simeq 0.95 \tau$ at $\phi=3 / C$. Thus the system undergoes a fast cascade of microcracks until the final break up for $\phi>\phi_{\text {break }}=3 / C$ :

$$
\phi_{\text {break }}=\frac{3 K T}{f_{o}\left(1-f_{o}\right)}=\frac{3\left(1-f_{o}\right)}{2 f_{o}\left(\ln \tau-0.5 \ln \frac{2 \pi K T}{f_{o}^{2}}\right)}
$$

This equation verifies that $\phi<<1$ for any value of $f_{o}$ if $K T$ is small enough. 


\section{B The disordered case}

In the disordered case the breakdown threshold $f_{c}$ of fibers is a random variable. Let $G\left(f_{c}\right)$ be the distribution of thresholds, that is $\int_{\eta}^{\infty} G\left(f_{c}\right) d f_{c}$ is the fraction of fiber with threshold bigger than $\eta$. The crucial point is that the distribution of $f_{c}$ evolves with time (i.e. $\phi$ ) as fibers break, in such a way that:

$$
\int_{0}^{\infty} G\left(f_{c}, \phi\right) d f_{c}=1-\phi
$$

The expected fraction of fibers which break at a given time $t$ is:

$$
R_{\phi}=\int_{0}^{\infty} P\left(f_{c}-\frac{f_{o}}{1-\phi}\right) G\left(f_{c}, \phi\right) d f_{c}
$$

We use the definition of $P(\eta)$ (see eq.A.1) to write:

$$
\begin{aligned}
R_{\phi} & =\int_{0}^{\infty} \int_{\left(\xi-f_{o}^{\prime}\right)}^{\infty} G_{T}(x) G(\xi, \phi) d \xi d x \\
& =\int_{0}^{\infty} \int_{\xi}^{\infty} G_{T}\left(f_{c}-f_{o}^{\prime}\right) G(\xi, \phi) d \xi d f_{c}
\end{aligned}
$$

where $f_{o}^{\prime}=\frac{f_{o}}{1-\phi}$.

The function $\psi\left(f_{c}, \xi\right)=G_{T}\left(f_{c}-f_{o}^{\prime}\right) G(\xi, \phi)$ is integrated in the plane $\left(f_{c}, \xi\right)$ within the region $R e$ which is defined by the intervals $\xi \leq f_{c}<\infty$ and $0 \leq \xi<$ $\infty$. In eq.(B.3) one may invert the order of the integration by considering that the intervals defining region $R e$ are: $0 \leq \xi<f_{c}$ and $0 \leq f_{c}<\infty$. Therefore eq. B.3. becomes:

$$
R_{\phi}=\int_{0}^{\infty} G_{T}\left(f_{c}-\frac{f_{o}}{1-\phi}\right) d f_{c} \int_{0}^{f_{c}} G(\xi, \phi) d \xi .
$$

Thus in the disordered case one may turn eq. A.10) into:

$$
\frac{d \phi}{d t}=\int_{0}^{\infty} G_{T}\left(f_{c}-\frac{f_{o}}{1-\phi}\right) d f_{c} \int_{0}^{f_{c}} G(\xi, \phi) d \xi
$$

with the condition imposed by eq.B.1. Notice that if the a delta distribution with normalization B.1 is chosen for $G$ then one gets from eq. B.5 the equation for the homogeneous case eq.(A.10). Equation eq. B.5 is far too complex to be integrated analytically. However one can greatly simplify this framework by assuming that the dynamics is controlled by the weakest fibers which are the first to break. This hypothesis can been checked in a direct numerical simulation using for $G_{0}\left(f_{c}\right)$ a Gaussian and an uniform distribution centered at $f_{c}=1$. The result of the numerical simulation are shown in fig.11 for the 
Gaussian distribution. We clearly see that are the weakest fibers which break first and that the distribution evolves just on the left side.

Starting from this numerical evidence of the behaviour of $G\left(f_{c}\right)$ one can find an approximated analytical solution of eq.B.5.

\section{B.1 Case of the normal distribution for thresholds}

Let the initial distribution $G_{0}\left(f_{c}\right)$ of thresholds be a normal distribution of variance $k T_{d}=\sigma_{o}^{2} / 2$ and mean value $\left\langle f_{c}\right\rangle=1$. In fig.11 we see that ( for $\left.2 \sqrt{2 K T}<\left(1-f_{o}\right)\right)$ when $\phi$ increases the distribution is deformed on the left side, whereas the right side remains unchanged. On a first approximation one can take into account this behaviour by splitting the distribution at time $t$ :

$$
G\left(f_{c}\right)=\frac{1}{\sqrt{\pi} \sigma_{o}} \exp \left(-\frac{\left(f_{c}-1\right)^{2}}{\sigma_{o}^{2}}\right) \quad \text { for } f_{c}>1
$$

and

$$
G\left(f_{c}\right)=\frac{1}{\sqrt{\pi} \sigma_{o}} \exp \left(-\frac{\left(f_{c}-1\right)^{2}}{\sigma_{d}^{2}}\right) \quad \text { for } f_{c}<1
$$

where $\sigma_{d}$ depends on $\phi$. The normalization condition B.1 yields $\sigma_{d}=\sigma_{o}(1-$ $2 \phi$ ), which roughly agrees with the numerical data. Thus the integral in eq.B.4 becomes

$$
\begin{array}{r}
R_{\phi}=\frac{1}{\sqrt{\pi}} \int_{0}^{1} \exp \left(-\frac{\left(f_{c}-\frac{f_{o}}{1-\phi}\right)^{2}}{\sigma^{2}}\right)\left(1-\operatorname{erf}\left(\frac{1-f_{c}}{\sigma_{d}}\right)\right) \frac{\sigma_{d} d f_{c}}{2 \sigma_{o} \sigma}+ \\
\frac{1}{\sqrt{\pi}} \int_{1}^{\infty} \exp \left(-\frac{\left(f_{c}-\frac{f_{o}}{1-\phi}\right)^{2}}{\sigma^{2}}\right)\left(\frac{\sigma_{d}}{\sigma_{o}}+\operatorname{erf}\left(\frac{f_{c}-1}{\sigma_{o}}\right)\right) \frac{d f_{c}}{2 \sigma}
\end{array}
$$

In order to find an analytical expression for eq. B.8 one has to consider that in the case of a Gaussian distribution $90 \%$ of the unbroken fibers have a threshold $f_{c}>f_{\min }=\left(1-\sqrt{\pi} \sigma_{d} / 2\right)$. One can make the hypothesis that only these fibers, with a large threshold, play an important role in supporting the applied

load. The mathematical counter part of this hypothesis is that only the part of the distribution with $f_{c}>f_{\min }$ has to be taken into account. Thus in the first integral of eq.B.8 the lower integration limit becomes $f_{\min }=\left(1-\sqrt{\pi} \sigma_{d} / 2\right)=$ $\left[1-\sqrt{\pi} \sigma_{o}(1 / 2-\phi)\right]$ and we can use $\operatorname{erf}(x) \simeq 2 x / \sqrt{\pi}$. After applying these approximations one gets:

$$
\begin{array}{r}
R_{\phi}=\frac{1}{\sqrt{\pi}} \int_{f_{\text {min }}}^{1+\frac{\sqrt{\pi} \sigma_{o}}{2}} \exp \left(-\frac{\left(f_{c}-\frac{f_{o}}{1-\phi}\right)^{2}}{\sigma^{2}}\right)\left(\frac{f_{c}-f_{\min }}{\sigma_{o} \sqrt{\pi}}\right) \frac{d f_{c}}{\sigma}+ \\
\frac{1}{\sqrt{\pi}} \int_{1+\frac{\sqrt{\pi} \sigma_{o}}{2}}^{\infty} \exp \left(-\frac{\left(f_{c}-\frac{f_{o}}{1-\phi}\right)^{2}}{\sigma^{2}}\right)(1-\phi) \frac{d f_{c}}{\sigma}
\end{array}
$$


Taking into account that,within the integration limits of the first integral, $\left(f_{c}-f_{\text {min }}\right)$ is almost constant with respect to the variation of $\exp \left(-\frac{\left(f_{c}-\frac{f_{o}}{1-\phi}\right)^{2}}{\sigma^{2}}\right)$, one can take the mean value of $f_{c}-f_{\min }$. Therefore one obtains the following approximated result:

$$
R_{\phi}=\frac{1-\phi}{2}\left[2-\operatorname{erf}\left(\frac{f_{\min }-\frac{f_{o}}{1-\phi}}{\sigma}\right)-\operatorname{erf}\left(\frac{1+\frac{\sqrt{\pi} \sigma_{o}}{2}-\frac{f_{o}}{1-\phi}}{\sigma}\right)\right]
$$

In this equation one can use the asymptotic approximation of the error function $\operatorname{erf}(x) \simeq 1-\exp \left(-x^{2}\right) /(x \sqrt{\pi})$. Thus one gets the equation describing the time evolution of $\phi$.

$$
\frac{d \phi}{d t} \simeq B_{n}(1-\phi) \exp \left(A_{n} \phi\right)
$$

where the coefficients $A_{n}$ and $B_{n}$ are the following:

$$
\begin{array}{r}
A_{n}=\frac{\left(1-\frac{\sqrt{\pi} \sigma_{o}}{2}-f_{o}\right)\left(f_{o}-\sqrt{\pi} \sigma_{o}\right)}{k T} \\
B_{n}=\sqrt{\frac{2 \pi}{k T}} \frac{\left(1+\exp \left(-\frac{\sqrt{\pi} \sigma_{d}(1-f)}{k T}\right)\right.}{2\left(1-\frac{\sqrt{\pi} \sigma_{o}}{2}-f_{o}\right)} \times \\
\exp \left(-\frac{\left(1-\frac{\sqrt{\pi} \sigma_{o}}{2}-f_{o}\right)^{2}}{2 k T}\right)
\end{array}
$$

Following the same procedure used in the homogeneous case, one finds that the failure time is:

$$
\begin{aligned}
\tau \simeq & \frac{2 \sqrt{2 \pi k T}}{\left(f_{o}-\sqrt{\pi} \sigma_{o}\right)\left(1+\exp \left(-\frac{\sqrt{\pi} \sigma_{d}(1-f)}{k T}\right)\right)} \times \\
& \quad \exp \left[\frac{\left(1-f_{o}\right)^{2}}{2 k T} \cdot\left(1-\frac{\sqrt{\pi} \sigma_{o}}{2\left(1-f_{o}\right)}\right)^{2}\right]
\end{aligned}
$$

Notice that for $\sigma_{d} \rightarrow 0$ one recover eq.A.17 for the life time in the homogeneous case. In section (4) we have seen that the $\tau$ predicted by eq. B.14) agrees very well with the results of the numerical simulations.

\section{B.2 The uniform distribution}

The same calculation can be done taking for $G\left(f_{c}\right)$ in eq.B.1 a uniform distribution of mean value $\left\langle f_{c}\right\rangle=1$ and width $D$. We find in this case that: 


$$
\begin{array}{r}
\tau \simeq \frac{\sqrt{2 \pi k T} 2}{\left(f_{o}-0.8 * D\right)\left(1+\exp \left(-\frac{0.8 D\left(1-f_{o}\right)}{k T}\right)\right)} \times \\
\quad \exp \left[\frac{\left(1-f_{o}\right)^{2}}{2 k T} \cdot\left(1-\frac{0.8 D}{2\left(1-f_{o}\right)}\right)^{2}\right]
\end{array}
$$

where the factor $0.8 D$ comes from the fact that in the case of the uniform distribution $90 \%$ of the the unbroken bonds have $f_{c}>f_{\min }(1-0.8 \mathrm{D} / 2)$.

\section{Discussion}

One observes that in both cases of uniform and normal distributions, the presence of a disorder on thresholds have the same effect over the general behaviour of the system. In particular one sees that disorder enhances the effect of thermal noise, in that it decreases in a multiplicative way the exponents in B.14 and B.15. We stress that thermal noise and disorder play different roles: indeed it is the thermal noise which drives the cracking process, while the disorder just enhances its effect. The effective temperature of eq. 16 is quite different from the result recently obtained by Roux. He finds that the effective temperature is the

sum of $K T$ and $K T_{d}$. Indeed our result is not directly comparable with that of Roux because he estimates the time needed to cut the first fibers. To compute this time Roux considers that the threshold distribution is almost quenched. This is certainly correct at the very beginning of the dynamics but it is not at longer times as we have shown in previous sections. In any case the time needed to cut the first fibers is negligible with respect to the total life time of the bundle if $\left(1-f_{o}\right)>\sqrt{2 K T_{d}}$. Furthermore the number of bonds which have the threshold in the tails of the distribution does not affect the resistance of the system, for example in the case of the Gaussian only $2 \%$ of the bonds have the threshold in the tail and in the case of a uniform distribution there are no tails. 


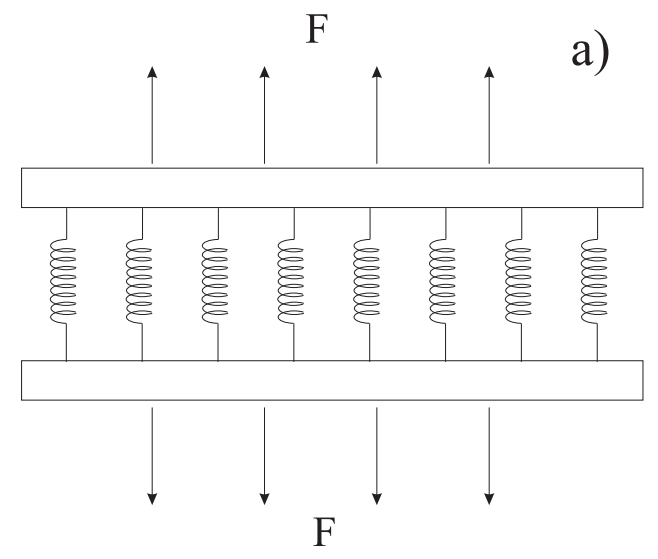

b)

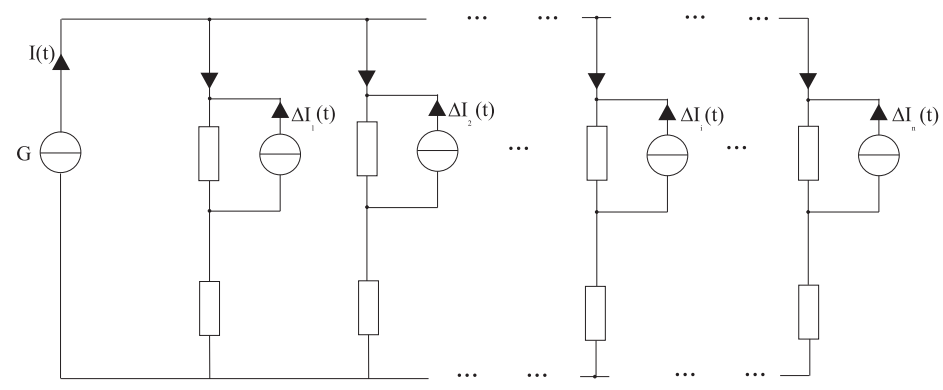

Figure 1: a) Modified DFBM: $N$ fiber in parallel, with the edges fixed on a rigid support are subjected to an externally imposed force $F$ which is distributed democratically on the net, that is all fibers (not broken) are affected in the same way. Each fiber is also subjected to a random (zero mean, normally distributed), addictive force $\Delta f_{i}(t)$, where is intended that $\Delta f_{i}(t)$ is a realization of a white, time independent stochastic process. Name $n(t)$ the number of broken fibers at time $t$; we derive the following expression of local force $f_{i}$ for the i-th fiber: $f_{i}=\frac{F}{N-n(t)}+\Delta f_{i}$. b) The equivalent of the fiber bundle model is the fuse network. One can think to our model as a fuse network where the Nyquist noise of several resistances (current generators $\delta I_{i}$ ) are the noise generators for each bond 
(a)

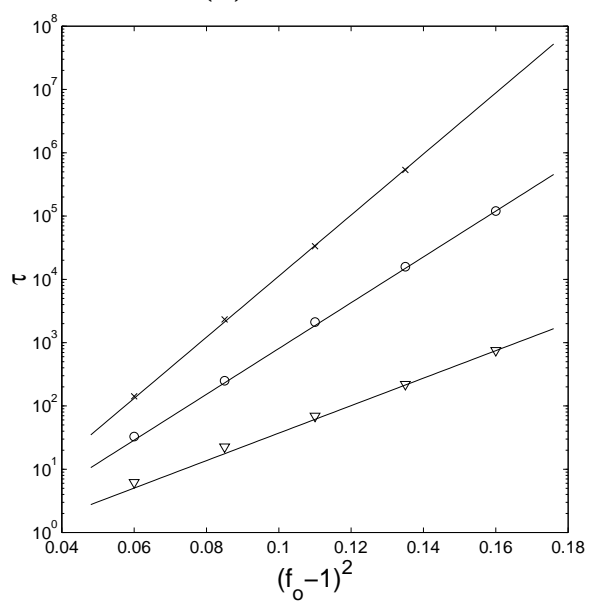

(b)

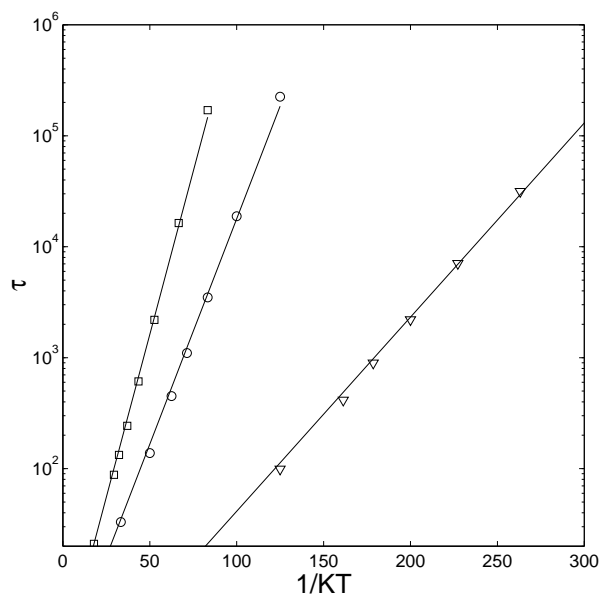

(c)

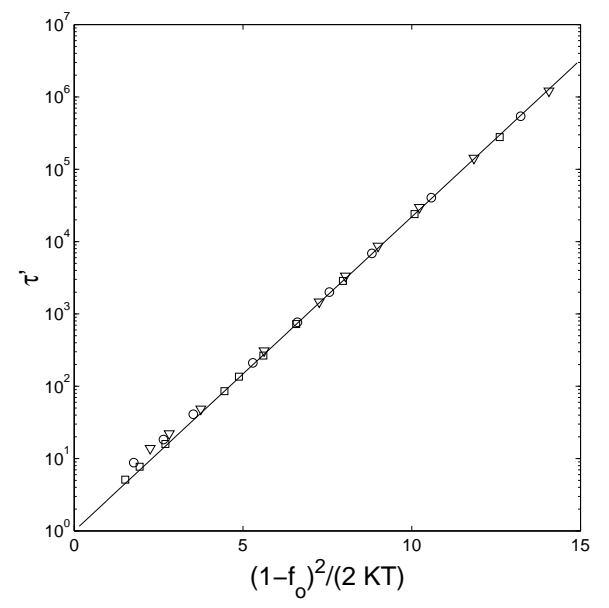

Figure 2: Homogeneous net $\left(K T_{d}=0\right)$ in a creep test. (a) Failure time $\tau$ as a function of $\left(1-f_{o}\right)^{2}$ for several values of the thermal noise variance $K T$ $((\times), K T=0.0045,(\circ), K T=0.006,(\nabla), K T=0.01)$. The continuous lines are the fits with eq.18 (b) $\tau$ as a function of $1 / K T$ for several values of imposed force $f \cdot N$. ( $\square) f_{o}=0.45 ;(\circ) f_{o}=0.54 ;(\nabla) f_{o}=0.7$. The continuous lines are the fits with eq.19. (c) $\tau^{\prime}=\tau f(2 \pi K T)^{-1 / 2}$ as function of $(1-f)^{2} /(2 K T)$. The same symbols of (b) are used. The continuous line is obtained from eq.13. 


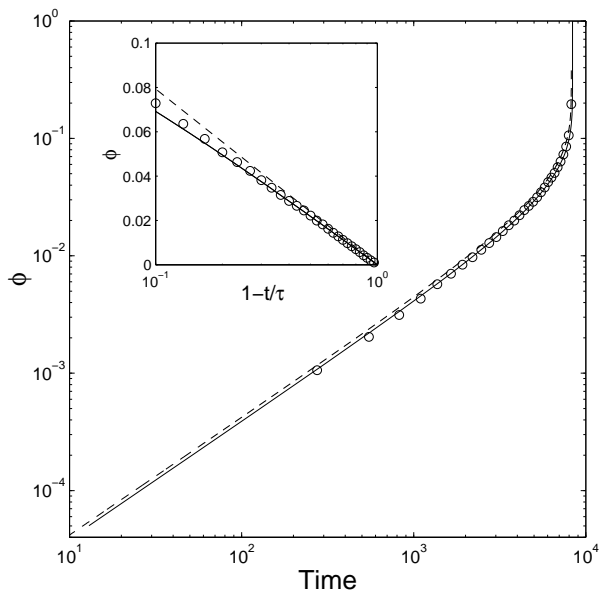

Figure 3: Time evolution of $\phi$ at $f=0.6, K T=0.008$ and $K T_{d}=0$. The continuous line corresponds to a solution obtained by the numerical solution of eq.12. The dashed line is instead the approximated solution (eq.14) of eq.12. The symbols (o) correspond to the results of the direct numerical simulation of the DFBM. These data are plotted in the inset as a function of $1-t / \tau$ in a semilog scale.

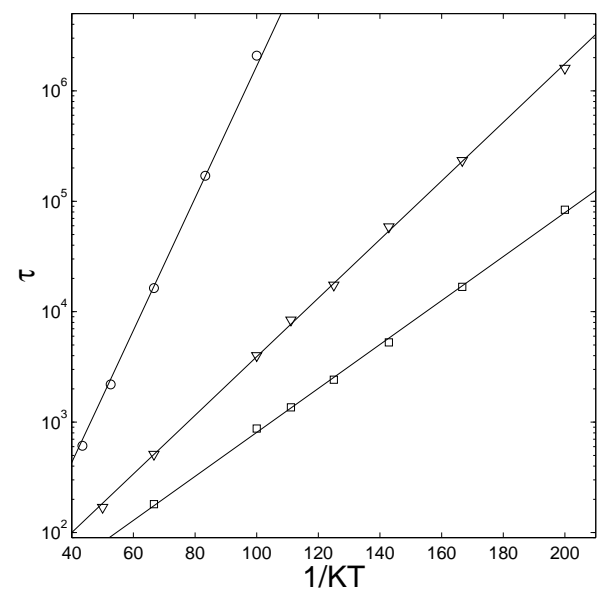

Figure 4: Heterogeneous bundle $\left(K T_{d} \neq 0\right)$. Dependence of the lifetime $\tau$ on $1 / K T$ at $f_{o}=0.45$. The different symbols correspond to different values of $K T_{d}:(\circ) K T_{d}=0,(\triangleleft) K T_{d}=0.02,(\square) K T_{d}=0.04$ Notice that both $\tau$ and $\frac{d \tau}{d K T}$ decrease as $K T_{d}$ increases, that is the more media become heterogeneous, the smaller is $\tau$ and the dependence on $K T$ of $\tau$. 


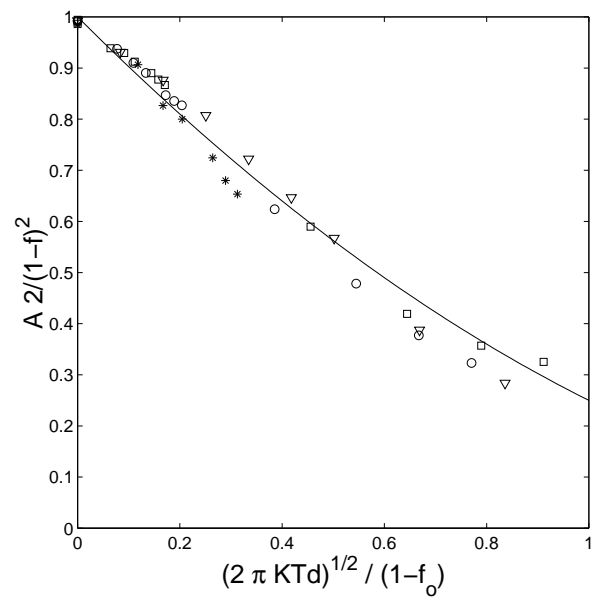

Figure 5: Dependence of $2 A /\left(1-f_{o}\right)^{2}$ ( $A$ is defined in equation 20) as a function of $\sqrt{2 \pi K T_{d}} /\left(1-f_{o}\right)$ for different values of $f_{o}$ : ( $\left.\square\right) f_{o}=0.45 ;$ ( $\left.\circ\right) f_{o}=$ $0.54 ;(*) f_{o}=0.7$. The continuous line is the theoretical prediction obtained from eq.20. The symbols $(\nabla)$ correspond to a numerical simulation done with uniform distribution at $f_{o}=0.54$. In this case the $\mathrm{x}$ axis is $0.8 D /\left(1-f_{o}\right)$ ( see appendix B.2).

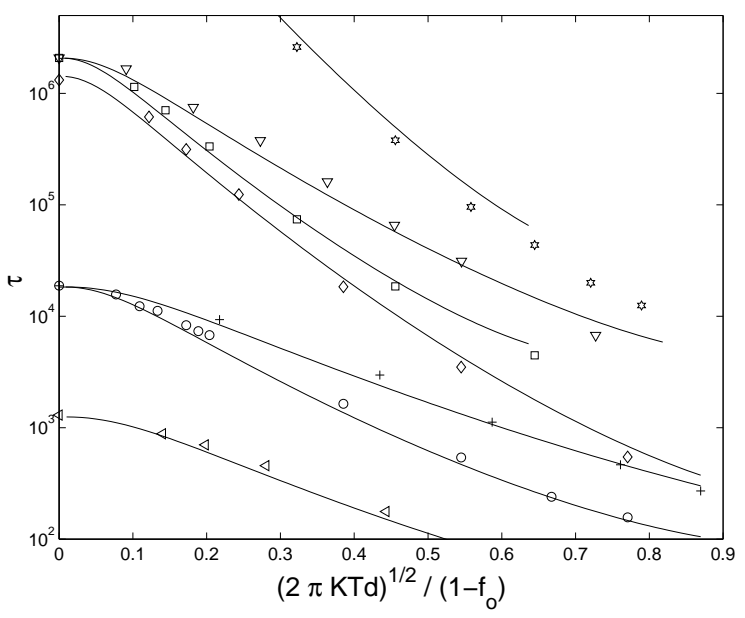

Figure 6: The lifetime $\tau$ is plotted as a function of the disorder noise variance $K T_{d}$ for several values of $K T$ and of $f_{o}$ and different threshold distributions. The continuous lines represent predictions of eq.15 for the Gaussian distribution and eq.B.15 for the uniform distribution. Discrete points are the results of numerical measures. For the Gaussian distribution: (०) KT = $0.01, f_{o}=0.54 ;(\square) K T=0.01, f_{o}=0.45 ;(\diamond) K T=0.007, f_{o}=0.54 ;(\triangleleft$ ) $K T=0.01, f_{o}=0.6 ;(\star) K T=0.0073, f_{o}=0.45$. For the uniform distribution $(+) K T=0.01, f_{o}=0.54 ;(\nabla) K T=0.01, f_{o}=0.45$. 

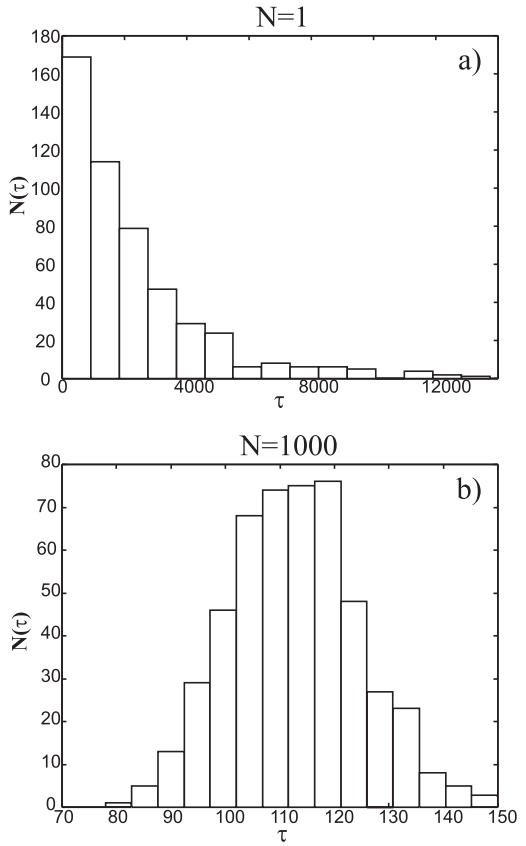

Figure 7: Lifetime distribution for a single fiber (a) and (b) for $N=1000$. $f_{o}=0.54$ and $K T=0.021$ in both cases

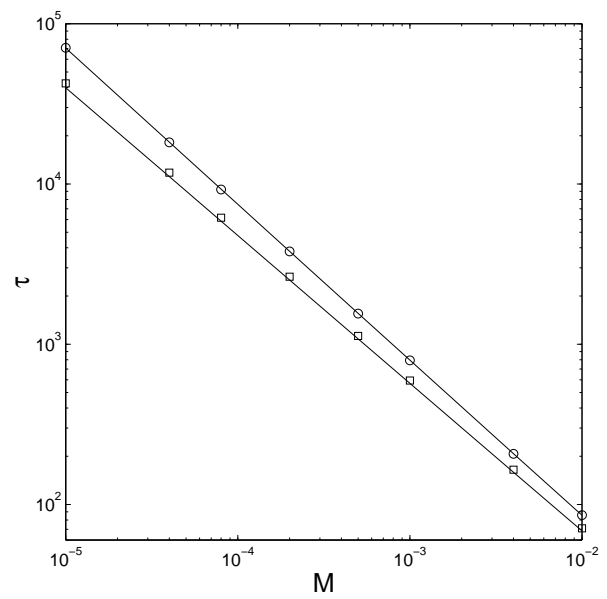

Figure 8: Failure time $\tau$ as a function of the slope $M$ in the case of a linear (in time) imposed force $F=M t$. The theoretical prediction of eq.22(continuous line) confirms the results obtained in numerical simulations. In these simulations: $N=1000, K T_{d}=0,(\circ) K T=0.005$ and $(\square) K T=0.02$ 


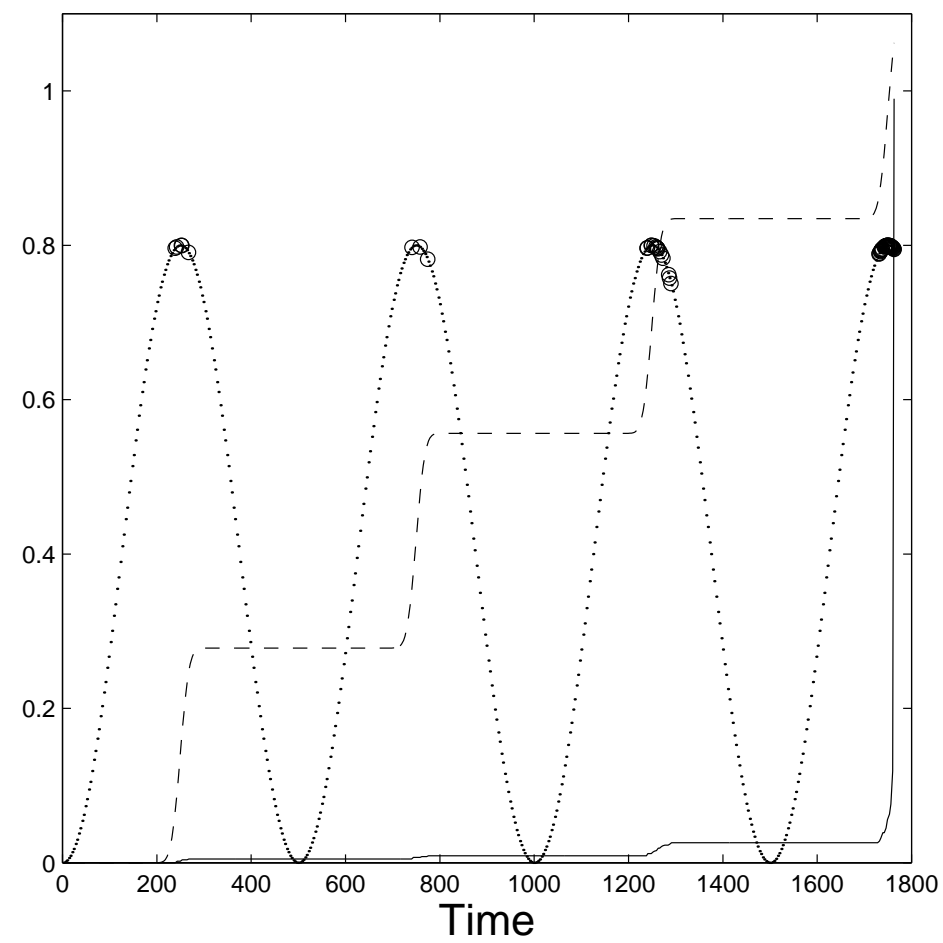

Figure 9: A sinusoidal force (dotted line) is applied to the bundle. The dashed line represents the numerical integration of the integral in eq.21. The circles indicate the occurrence of breaking events. Finally the continuous line is the fraction of broken fiber $\phi$ as measured by the direct numerical simulation of the fiber bundle. The time predicted by eq.21 perfectly agrees with the that of the direct numerical simulation. In this simulation $N=1000, K T_{d}=0$, $K T=0.003$ 

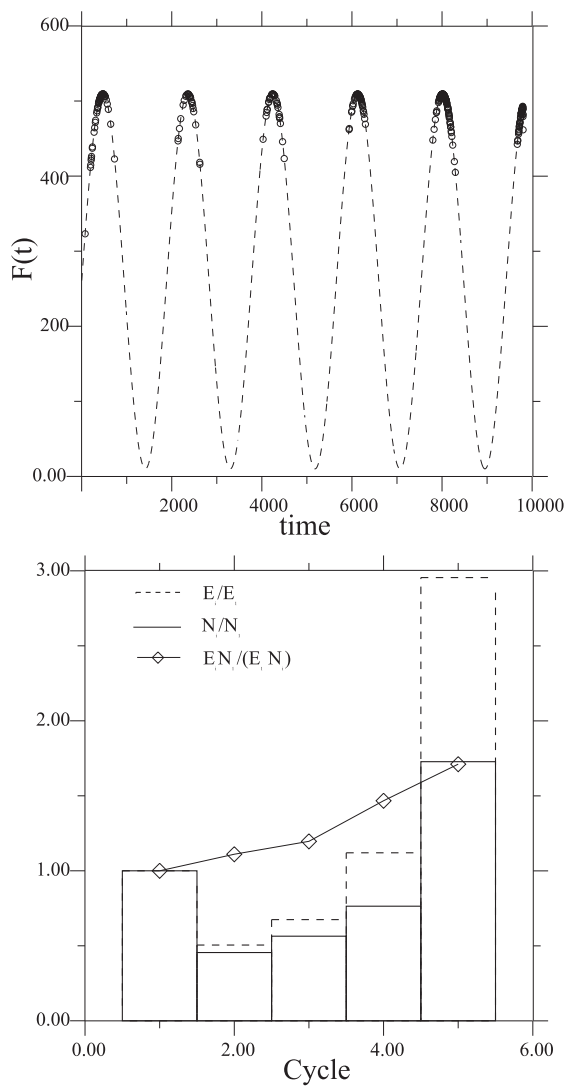

Figure 10: (a) Imposed force $F=F_{o}\left[1-\cos \left(\frac{2 \pi}{T_{\omega}} t\right)\right]$ as a function of time in a single realization; discrete points represent the occurrence of breaking events. (b) Number of events $N_{i}$ (continuous bars) and the energy $E_{i}$ (dashed bars) released during the i-th cycles, as functions of $i$. Both these quantities are normalized to the values of the first cycle. Discrete points represent the normalized mean energy $\frac{E_{i} / N_{i}}{E_{1} / N_{1}}$ of an event occurred during the i-th cycle. We observe that Kaiser effect is not valid, that is there are a large number of events after the first cycle. The parameters of this simulation are $N=1000$ and $K T=9 \cdot 10^{-3}$ $K T_{d}=1.5 \cdot 10^{-2}$. 
(a)

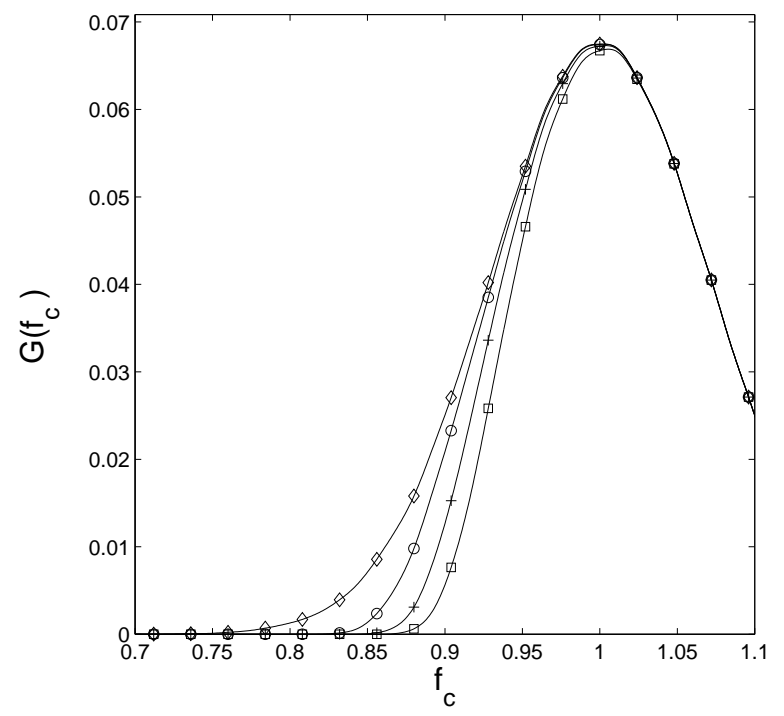

(b)

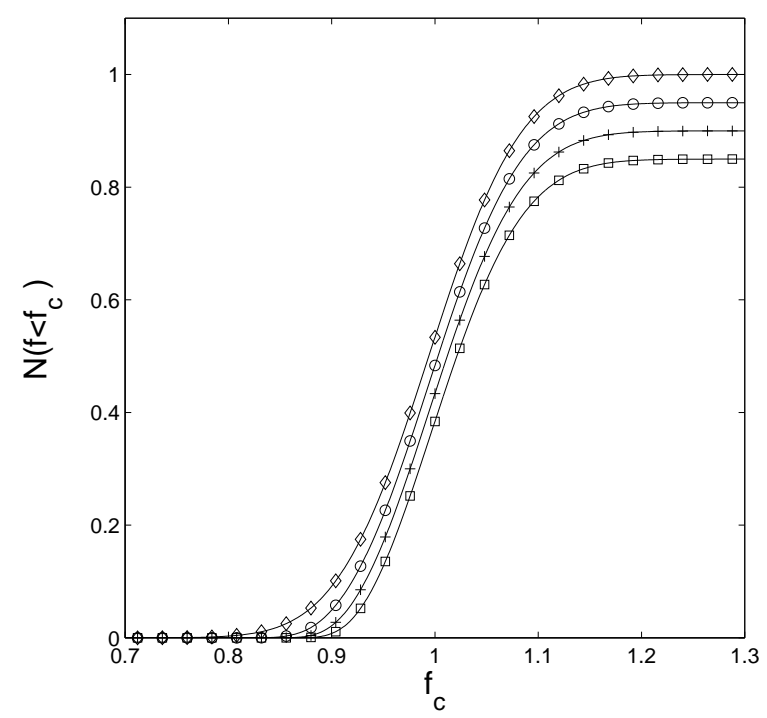

Figure 11: Heterogeneous net. Evolution as a function of $\phi$ of the threshold distribution at $f=0.6, K T=0.006$ and $K T d=0.005$. (a) Evolution of $G\left(f_{c}\right)$ at $\phi=0(\diamond), \phi=0.05(\circ), \phi=0.1(+)$ and $\phi=0.15(\square)$. The fraction $\phi=0.15$ corresponds to $t \simeq 0.9 \tau$. (b) Evolution of the integral distribution $N\left(f<f_{c}\right)=\int_{0}^{f_{c}} G(x) d x$ at the same $\phi$ used in (a). 


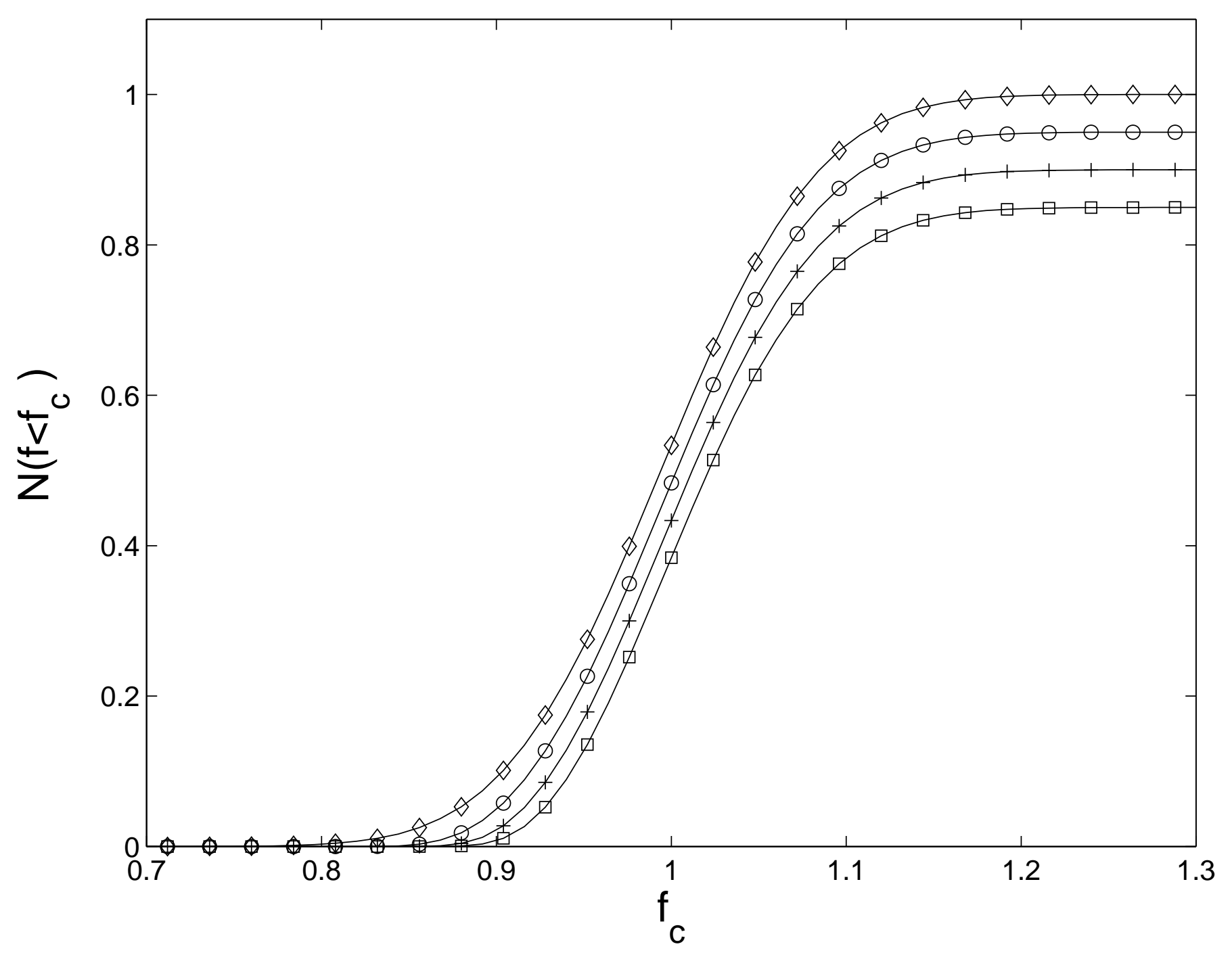




\section{Answer to referee A:}

We thank the referee for his useful comments. The manuscript has been changed in order to keep them into account. Specifically the answers to the questions of the referee are the followings:

0 ) We agree the abstract contained too strong statements. It has been changed.

1) All these suggestions have been taking into account

2) We thank the referee for having indicated these references which were unknown to us. References to the work of Coleman and Phoenix have been added.

3) We agree the statement has been changed.

4) We agree. The validity limits of our approximation are now clearly indicated in the text.

5) We added the symbol for $f=0.7$ in the figure.

6) These changes have been done.

7) We agree. The text has been corrected.

8) The suggestion has been taken into account.

9) We agree is the fraction of fiber. It has been changed.

10) Now we explain how to go from eq.B2 to eq.B3 (now eq.B4).

11) We agree with the referee that our assumptions were badly explained in the previous manuscript. This part has been completely rewritten. We agree that our approximations do not work for large temperature and this is now clearly indicated. However we disagree with the referee on two points. The fact that the threshold distribution is not quenched and that the fibers with threshold larger than one are not broken are not assumptions but they come from a numerical evidence. We use this evidence in order to find an analytical formula which works for the cases we have analyzed. Furthermore the $90 \%$ has nothing to do with temperature because it is reasonable to think that the most important fibers are those with the largest thresholds. Indeed this approximations works quite well also for the uniform distribution which has no tails. From this point of view there is no reason to believe that our approximation fails for $T$ going to zero. However we completely agree with the referee on another point. The comparison with the results of Roux's paper was incorrect and imprecise. The discussions in appendix $\mathrm{C}$ and in the conclusions of the paper have been changed. 


\section{Answer to referee B:}

We thank the referee for the careful reading of the manuscript. His suggestions have been taken into account and a figure with cycling loading has been added. The paper has been carefully read.

The comments of the referee allows us to make our point of view very clear.

The recent experimental results were the motivation of our work. We wanted to study the influence of disorder in the crack nucleation processes. The fiber bundle is a certainly a toy model which could be useful to understand what is going on. To make this point clear we changed the abstract and part of the introduction.

However we strongly disagree with the referee when he says that everything is clear and the only unexplained experiments are ours. We kindly explain the reasons: a) The temperature is very high in all the experiments.

In our experiment it is $3000 \mathrm{~K}$ In the Pauchard experiment (ref.14) the temperature is larger than $1000 \mathrm{~K}$. In the Bonn experiment (ref.15) it is larger than $10^{10} \mathrm{~K}$. The referee can easily check our claims taking the data of ref.14 (PRL of Pauchard et al.) and ref. 15 (Science, Bonn et al.) In order to make this point clear we wrote these temperatures in the introduction.

b) The value of the dimensionality d, measured in Bonn's paper, is obtained from fig. 4 of the paper on Science (ref.15). In this figure the vertical axis is the $\log$ of the log of the lifetime. However by making the second log the authors of ref.15 forgot that there is an additive constant (the $\log \left(\tau_{o}\right)$ ). If the calculation is done taking into account the $\log \left(\tau_{o}\right)$ (this can be approximately obtained using the values given in the text of ref.15) one gets (if a power law still exists) values of $\mathrm{d}$ which are very different from those claimed in ref.15. Thus there is something wrong in that paper.

c) The Pauchard data are much better fitted by the Mogi's law than the Pomeau's law. A simple chi-square test shows this. Further I want to recall to the referee that in that paper only the best three points have been chosen. "Best" means here the points which give the best fit on the Pomeau's law and not the points with the smallest statistical errors. This kind of data analysis can be found in a paper published on PRL.

d) Poissonian statistics are observed only by Bonn. In the other experiment the life time distributions are Gaussians.

We did not add points b) and c) in the introduction of our paper because it will generate an useless and nasty polemics with Bonn and Pauchard. We 
would like to avoid this polemic. However if the referee thinks that it is important to put the points b) and c) in the introduction of our paper, we are ready to do that. We think that this is not really a key point and it will make our introduction too long. We want just to kindly drive the attention of the referee on the fact that in spite of his nasty comments our experiment is the best verification of the Pomeau's law, as far as the force dependence is concerned. At this point the referee has two alternatives. Either he think that Pomeau's law is useless or that it is an extremely good idea but one has to improve the understanding of its applicability. This is exactly what we think and what we have done in our paper. We show that disorder can enhance the role of thermal noise at least in a toy model. Of course the activation energy of the fiber bundle is not the same of a continuous medium and we discuss this point in section 3.1. However the thermal activation of the fiber bundle seems to be already so reach which certainly merits to be studied. 


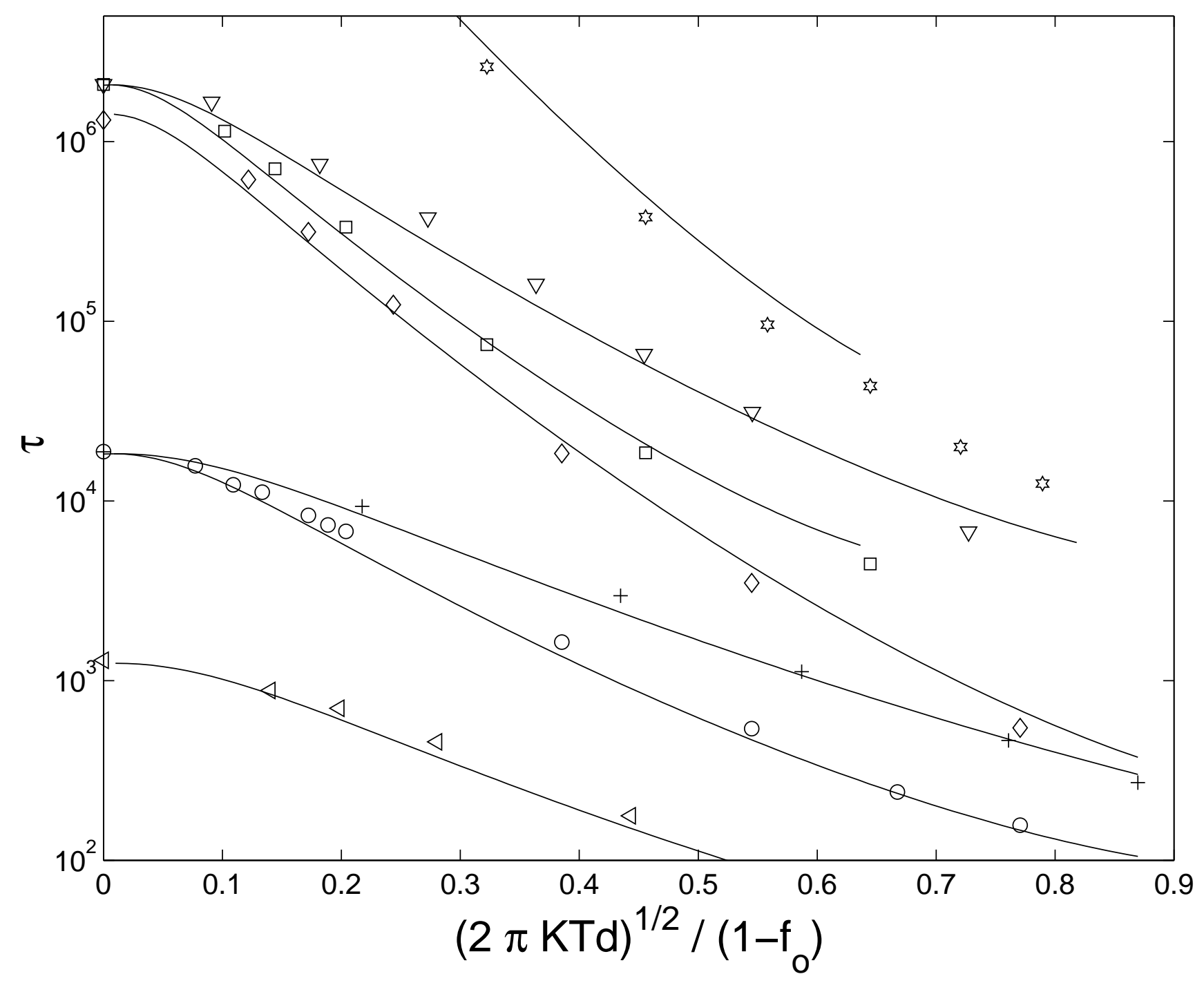




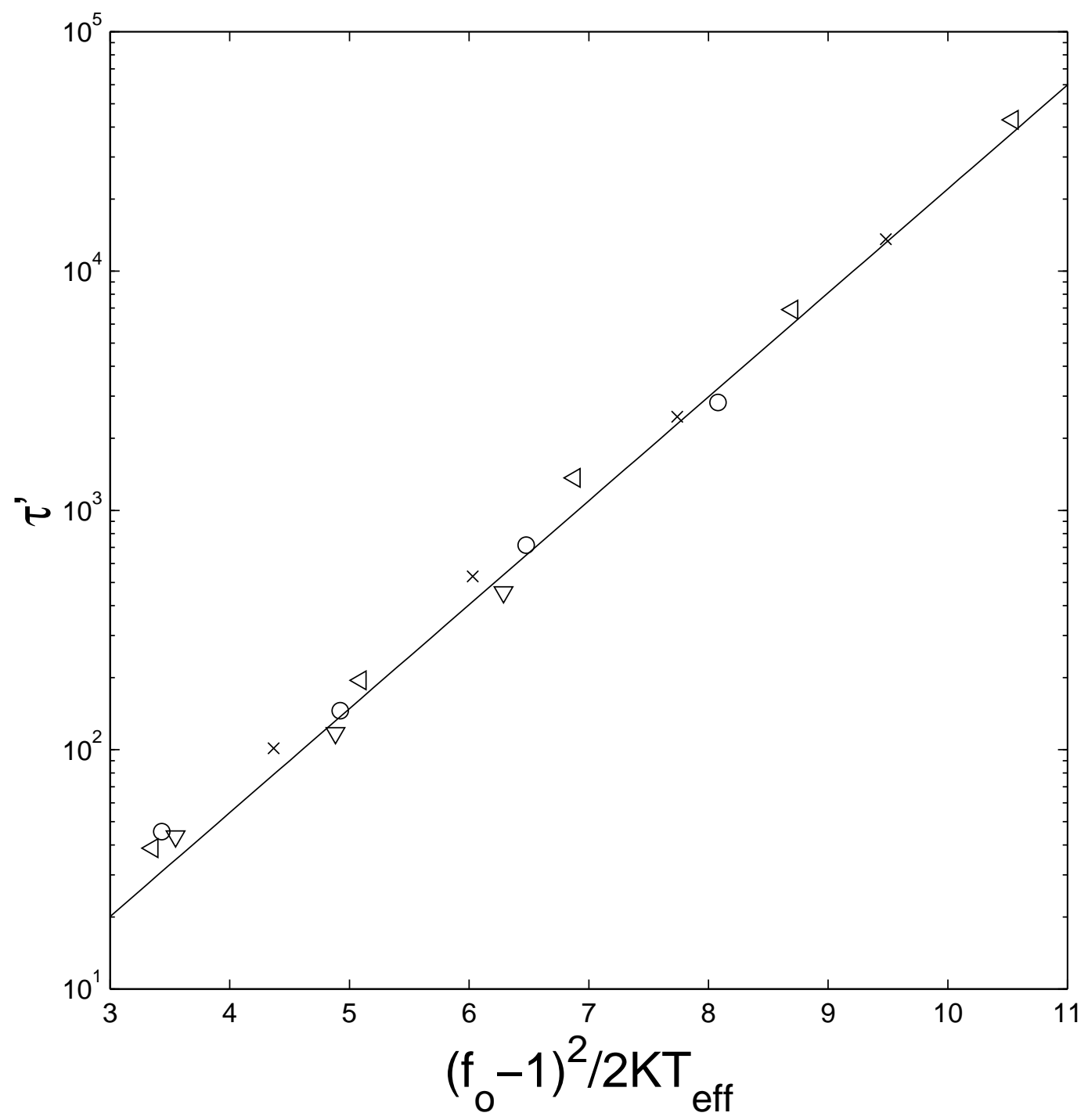

